\title{
Asthmatische Mütter neigen zur Frühgeburt
}

\author{
Eine große Studie zeigt, dass werdende Mütter mit Asthma ein höheres Risiko haben, ihr Kind zu \\ früh zu bekommen. Auch entstehen beim Nachwuchs häufiger bronchopulmonale Dysplasien.
}

_ Für die Studie wurde ein Register aller Geburten der Jahre 2007 bis 2010 in Kalifornien $(\mathrm{n}=2.009 .511)$ herangezogen. Es ging um drei Fragen:

- Ist die Prävalenz der Frühgeburtlichkeit bei schwangeren Müttern mit Asthma erhöht?

- In welchem Stadium der Schwangerschaft ist das Risiko am höchsten?

- Hängen die Prävalenzen von Frühgeburtlichkeit und bronchopulmonaler Dysplasie (BPD) bei Kindern asthmatischer Mütter zusammen?

Für die letzte Frage bildete man eine Kohorte, die über 90\% der Frühgeborenen einschloss, welche im Untersuchungszeitraum auf Neugeborenen-Intensivstationen behandelt wurden $(\mathrm{n}=21.944)$.
Anhand der Schwangerschaftsdauer wurden drei Gruppen von frühgeborenen Kindern gebildet: < 28 Wochen, 28 32 sowie 33-37 Wochen.

Für asthmatische Mütter ergab sich eine Prävalenz an Frühgeburten von $8,6 \%$, welche signifikant höher lag als die $5,4 \%$, die sich für Mütter ohne Asthma errechneten. Das höchste Risiko bei den asthmatischen Müttern lag in der Zeit zwischen der 28. und 32. Schwangerschaftswoche.

Die Prävalenz der Entwicklung einer BPD war unter den Frühgeborenen nicht höher als unter den normal Geborenen. Jedoch ergab eine entsprechende Stratifizierung ein signifikant höheres BPDRisiko für Kinder, deren Mütter in der
Schwangerschaft nicht auf ein Glukokortikoid eingestellt waren.

- Gage S, Kan P, Lee HC et al. Maternal Asthma, Preterm Birth, and Risk of Bronchopulmonary Dysplasia. J Pediatr. 2015;167:875-80.e1

\section{KOMMENTAR}

Die Studie zeigt, dass asthmatische Frauen während der Schwangerschaft einer besonderen Betreuung bedürfen, die eine sorgfältige Interaktion zwischen Geburtshilfe und betreuendem Arzt voraussetzt. Keineswegs darf auf eine gezielte und sorgfältig titrierte antiasthmatische Therapie verzichtet werden. Die Studie zeigt auch, wie wichtig es ist, werdende asthmatische Müttern mit Glukokortikoiden zu behandeln, um eine BPD bei ihren Kindern zu verhindern.

Prof. em. Dr. med. Dr. h. c. D. Reinhardt

\section{Ptosis nach einem Bad im Roten Meer}

Ein 52-jähriger Mann stellte sich mit Ptosis des linken Oberlides und eingeschränkter Beweglichkeit der Orbitamuskulatur am linken Auge vor (Abb. A). Vier Wochen zuvor hatte er nach einem Bad im Toten Meer eine Schwellung des

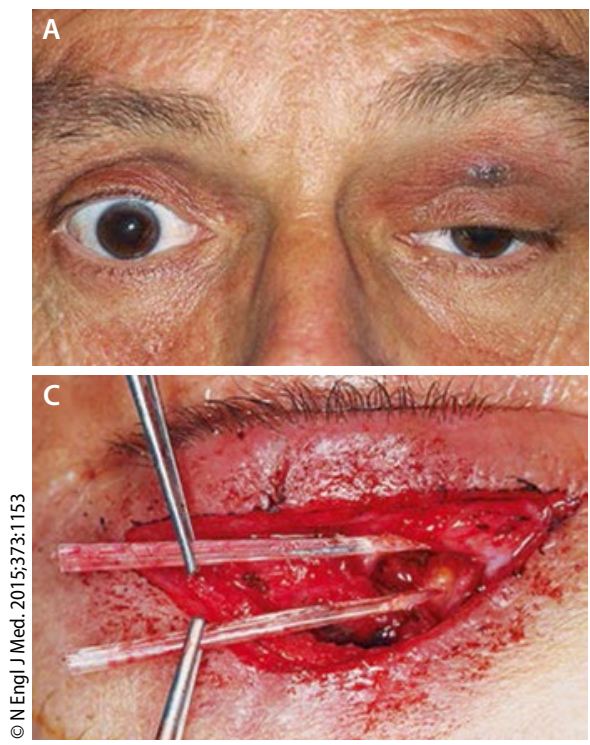

linken Oberlids bemerkt. Er war beim Schwimmen mit einem Fischschwarm in Kontakt gekommen. Im MRT zeigte sich ein Granulom im Oberlid und im vorderen und oberen Bereich der Orbita (Abb. B), allerdings kein Fremdkör-

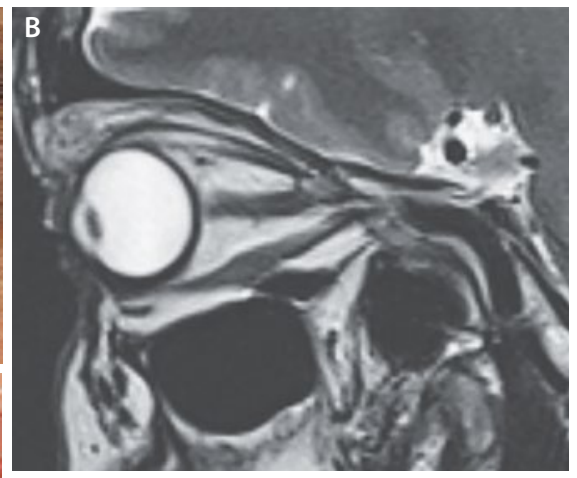

Ptosis des linken Oberlides (A), Granulom im Oberlid und im vorderen und oberen Bereich der Orbita im MRT (B), röhrenförmige Fremdkörper in der Augenhöhle (C). per. Bei der chirurgischen Eröffnung fand man allerdings zwei röhrenförmige, durchsichtige Fremdkörper, die entfernt wurden (Abb. C). Man musste annehmen, dass die nadelartigen Strukturen den M. levator palpebrae und den $M$. rectus superior fixiert und immobilisiert hatten.

Eine genauere Analyse der Fremdkörper durch einen Fischexperten ergab, dass es sich um Material vom Kiefer eines Halbschnäblers handelte. Halbschnäbler oder Halbschnabelhechte sind Fische aus der Ordnung der Hornhechtartigen, die sich bevorzugt in flachen und ufernahen Zonen tropischer Gewässer aufhalten, z. B. im Roten Meer. Ihr besonderes Kennzeichen ist der stark verlängerte Unterkiefer, der die Länge des Oberkiefers weit übertrifft. Offensichtlich hat sich ein Halbschnäbler seinen Unterkiefer am Auge des Patienten abgebrochen. Drei Monate nach der Operation hatten sich die Ptosis und die eingeschränkte Beweglichkeit der Orbita vollständig zurückgebildet.

Prof. Dr. med. H. S. FüeßI

- Lagrèze WA, SchaberM (wolf.lagreze@uniklinik-freiburg.de). Ptosis after swimming in the Red Sea. N Engl J Med. 2015;373:1153 